\title{
Theoretische Physik in den dreißiger Jahren
}

\author{
Die Entwicklung einer Wissenschaft unter ideologischen Zwangsbedingungen
}

Von Karl von Meyenn

Durch eine geschickte Wissenschaftspolitik, die auf dem Prinzip der Selbstverwaltung begründet war, und durch die starke mathematische Ausrichtung der exakten Naturwissenschaften, die besonders von Göttingen ausging, waren die theoretischen Physiker der Weimarer Republik trotz der allgemeinen Misere nach dem Ersten Weltkrieg in der Lage, äußerst erfolgreich zur Aufklärung der Atomrätsel beizutragen. Diese glanzvolle Epoche der theoretischen Physik, die mit der Aufstellung der Quantenmechanik ihren Höhepunkt erreichte, hat man deshalb auch als die der goldenen $20 \mathrm{er}$ Jahre bezeichnet.

Eine Entwicklung, die weder durch den Krieg noch durch die wirtschaftliche Notlage der Nachkriegsjahre aufgehalten werden konnte, kam dann innerhalb weniger Jahre durch die wissenschaftsfeindliche Politik der Nationalsozialisten völlig zum Erliegen. Arnold Sommerfeld, ein Physiker, der durch seine Forschung und Lehre im Zentrum dieser Entwicklung gestanden hatte, schrieb im September 1937 resigniert an Einstein: «Die Zukunft sieht trübe aus für die deutsche Physik. Ich muß mich damit trösten, daß ich ihr goldenes Zeitalter 1905-1930 tätig miterlebt habe.»

Begleitet von diesen Verfallserscheinungen in Deutschland und dem Aufschwung der Wissenschaft in Amerika und in Rußland, vollzog sich während der dreißiger Jahre auch ein grundlegender Wandel im Bereich der physikalischen Forschung selbst. Das Interesse verlagerte sich von der Hülle auf den Atomkern, von kleinen zu hohen und höchsten Energien. Das führte zu ganz neuartigen und aufwendigeren Untersuchungsmethoden und zu einem anderen Forschungsstil. Individualforschung wurde in zunehmendem Maße durch Teamarbeit ersetzt. Das Forschungslabor mußte jetzt immer mehr den Großforschungsanlagen Platz machen ${ }^{1}$. Doch dieser Wandel betraf in erster Linie die Experimentalphysik. Der Theoretiker konnte hingegen nach wie vor Wissenschaft in traditioneller Weise betreiben.

Und gerade dieses Gebiet war es, das sich Ende der zwanziger Jahre in Deutschland so vorzüglich entwickelt hatte. Aus den Schulen von Bohr, 
Sommerfeld und Born war eine neue Generation von hochtalentierten Theoretikern hervorgegangen, die jetzt danach strebte, weitere Pflanzstätten für ihre Disziplin einzurichten. Trotz der noch lange Zeit hindurch bestehenden Boykottmaßnahmen gegen deutsche Gelehrte durch die Wissenschaftler der Ententemächte setzte gerade bei den Theoretikern bald ein äußerst reger wissenschaftlicher Austausch mit dem Auslande ein. Neben den traditionsreichen Zentren in Berlin (Einstein, Planck und Schrödinger), München (Sommerfeld), Göttingen (Born) und Leipzig (Heisenberg und Hund) waren Kopenhagen (Bohr mit seinen Mitarbeitern), Cambridge (Dirac), Paris (Louis de Broglie), Leiden (Ehrenfest) und Zürich (Pauli und Wentzel) beliebte Studienplätze der jungen Nachwuchskräfte.

Auch die experimentelle Forschung in Deutschland war gut repräsentiert. Otto Stern und seine Mitarbeiter hatten in Hamburg mit dem Aufbau der weltbekannten Atom- und Molekularstrahlapparaturen begonnen ${ }^{2}$. Die Atom- und Kernspektroskopie wurde besonders durch die Gruppen von Hermann Schüler in Potsdam und Walter Bothe (seit 1932) in Heidelberg vorangetrieben. Ebenso war die Kernphysik in Berlin durch die enge Zusammenarbeit von Otto Hahn und Lise Meitner glänzend vertreten ${ }^{3}$. Auf dem Gebiete der Höhenstrahlung haben besonders die Messungen von Erich Regener in Stuttgart, von Werner Kolhörster in Berlin und von ihrem österreichischen Pionier Victor Hess in Graz die theoretische Bearbeitung dieser Phänomene ermöglicht ${ }^{4}$. Die überraschenden Ergebnisse bei tiefsten Temperaturen stellte die Theoretiker vor neue Rätsel ${ }^{5}$.

Auch die experimentelle Forschung dieser Jahre erhielt große Impulse aus der Theorie. Für die von vielen Theoretikern bisher gemiedene Festkörperphysik eröffneten sich mit Paulis Beitrag zur Theorie des Paramagnetismus der Metallelektronen ganz neue Perspektiven. Sommerfeld und seine Schüler entwickelten daraufhin eine quantentheoretische Elektronentheorie der Materie ${ }^{6}$.

Aufsehenerregende Entdeckungen und Hypothesen hatten zu Beginn der dreißiger Jahre immer mehr die Kernphysik in den Vordergrund gerückt: Walter Bothe und Herbert Becker sahen 1930 die Möglichkeit der Kernanregung ${ }^{7}$. Nur wenig später entdeckte Carl Anderson in der kosmischen Höhenstrahlung positive Elektronen, die der umstrittenen Diracschen Löchertheorie erneuten Auftrieb gaben ${ }^{8}$.

Noch wichtiger war eine zuerst von Joliot-Curie beobachtete neue Sekundärstrahlung beim Kernzerfall, die James Chadwick 1932 als Neutronenstrahlung identifizierte ${ }^{9}$. Diese Feststellung war für die Theorie des 


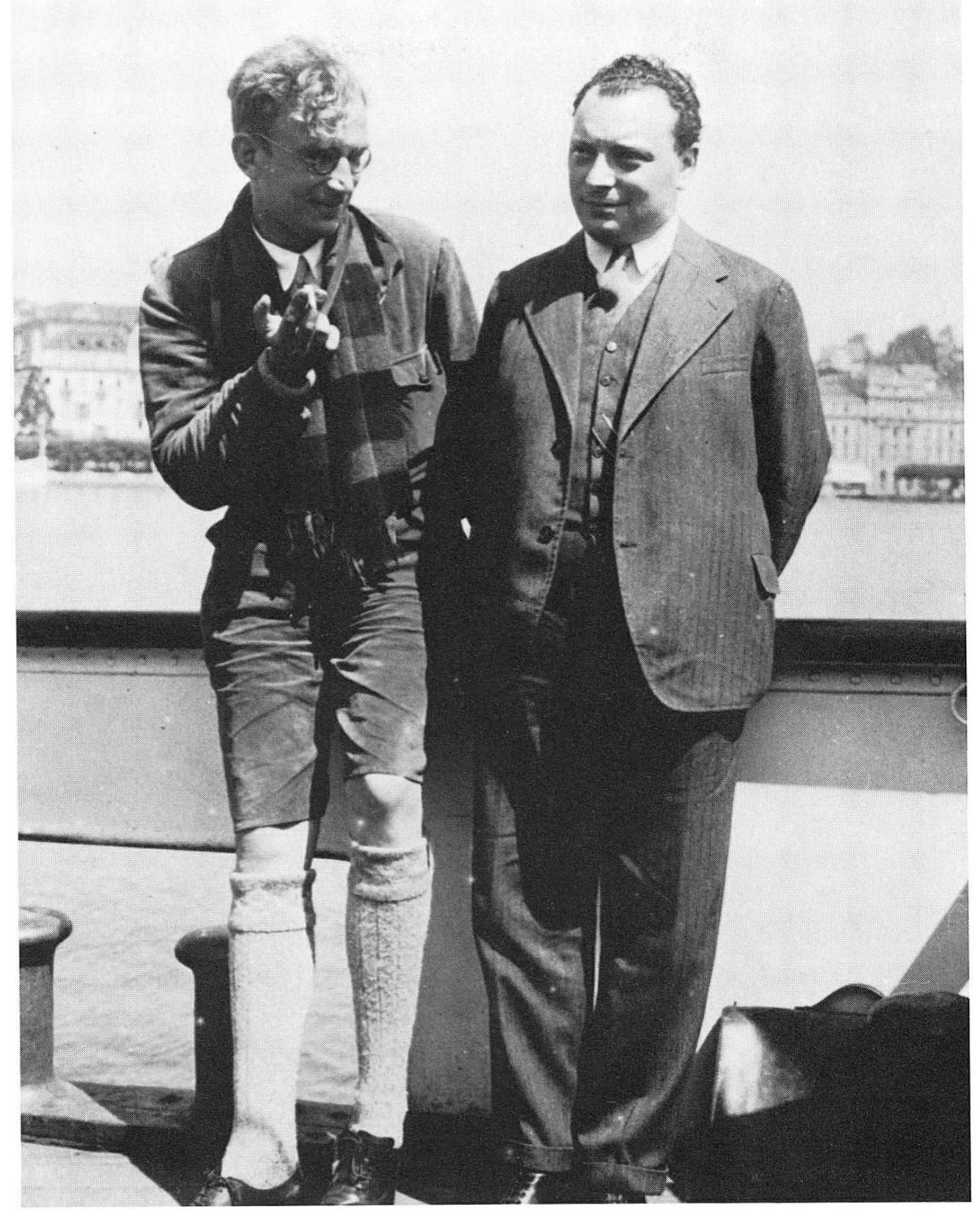

Abb.1. Pauli im Gespräch mit dem russischen Physiker George Gamow während einer Schiffsreise auf dem Genfersee 
Atomkerns von ähnlicher Bedeutung wie einst die Entdeckung des Elektrons, die zu einem Verständnis des Atomaufbaus geführt hatte. Wenig später publizierte Heisenberg die erste darauf fußende Theorie des Atomkerns ${ }^{10}$.

Eine Sonderstellung schließlich nimmt die Forderung eines hypothetischen Teilchens, des Neutrinos, zur Erklärung des mysteriösen $\beta$-Zerfalls durch Pauli ein, da sie zu vielen theoretischen Spekulationen anregte, die sich später für das Verständnis der Kernkräfte als sehr fruchtbar erwiesen ${ }^{11}$.

Aber auch in Amerika, Rußland und Japan hatte man seit den frühen 20er Jahren begonnen, verstärkt die theoretische Richtung in der Physik zu entwickeln.

In einem Brief vom 24. Januar 1921 an den soeben nach Pasadena gezogenen Kollegen Paul Epstein schreibt Born: «In experimenteller Hinsicht waren die Amerikaner uns schon lange gleichwertig, und in manchem überlegen. Jetzt wird auch die Theorie dort zum Leben erwachen, da Sie die Führung übernehmen.» ${ }^{12}$

Außer Epstein waren es von 1923-1927 hauptsächlich der Bohr-Schüler Oskar Klein, die beiden Ehrenfest-Schüler Uhlenbeck und Goudsmit und der Sommerfeld-Schüler Otto Laporte, welche in Ann Arbor diese Bestrebungen energisch förderten ${ }^{13}$. Eine weitere Stütze der theoretischen Physik in Amerika wurde der Münchener Physiker Karl Herzfeld, den man 1926 an die Johns Hopkins University berief. Der bekannte Tübinger Theoretiker Alfred Landé nahm 1931 einen Ruf an die Columbus-University in Ohio $a^{14}$. Bis 1931 hatten sich über 15 europäische Physiker in den Vereinigten Staaten niedergelassen ${ }^{15}$.

Zudem besuchten während der Jahre 1919-1932 etwa fünfzig jüngere amerikanische Physiker europäische Universitäten und Forschungseinrichtungen und nahmen vorwiegend theoretische Erfahrungen mit. Unter ihnen finden wir Oppenheimer ${ }^{16}$, Slater ${ }^{17}$, Rabi ${ }^{18}$, Breit ${ }^{19}$, Dennison ${ }^{20}$, Eckart $^{21}$, Feenberg ${ }^{22}$, Kemble ${ }^{23}$, Houston ${ }^{24}$, Kronig ${ }^{25}$, Mulliken ${ }^{26}$, Pauling ${ }^{27}$, van Vleck ${ }^{28}$, Kennard ${ }^{29}$, alles Namen, die dem Physiker wohlvertraut sind.

In Leningrad bildeten Landau, Ivanenko und Gamow den ersten Keim einer theoretisch orientierten Schule, die aufmerksam die Entwicklungen im Ausland verfolgte ${ }^{30}$. Staatliche Stipendien gestatteten vielen jungen sowjetischen Physikern einen Studienaufenthalt im westlichen Ausland. Unter ihnen finden wir bekannte russische Gelehrte wie Landau, Frenkel, Gamow und Fock. 
Ebenso besuchten japanische und indische Physiker häufig europäische Universitäten. Nishina und Sugiura haben sehr erfolgreich bei Bohr in Kopenhagen gearbeitet. Später, als Leipzig unter Heisenberg in einem guten Ruf stand, war Sinztiro Tomonaga dort zu Gast ${ }^{31}$.

Spätestens seit der Volta-Feier im Jahre 1927 in Como wurde die wissenschaftliche Welt auf Italien aufmerksam. Besonders die kernphysikalischen Arbeiten der Gruppe um Fermi führte in den folgenden Jahren dazu, daß nun auch Italien in das Netz der maßgeblichen Forschungsstätten einbezogen wurde, mit denen ein wissenschaftlicher Austausch stattfand ${ }^{32}$.

Obwohl dieses Netz schon damals sehr weitgefächert war, haben die beteiligten Forscher in sehr intensivem Kontakt miteinander gestanden. Der durch die rasche Entwicklung sich ständig ändernde Erkenntnisstand wurde auf diese Weise schnellstens weitergeleitet und durch Gegenüberstellung vereinheitlicht.

Neben dem Austausch von Mitarbeitern und den Vortragsreisen führender Gelehrter im In- und Ausland spielten hierfür auch die internationalen Konferenzen dieser Epoche eine überragende Rolle. Zu den Solvay-Kongressen, die jedesmal ein im Brennpunkt der Forschung stehendes Gebiet zum Gegenstand hatten, wurde nur eine kleine Gruppe erlesener Gäste nach Brüssel geladen. Eine Einladung zu diesen Veranstaltungen empfand man als eine hohe Auszeichnung und die publizierten Kongreßberichte enthielten viele Anregungen für die zukünftige Forschung ${ }^{33}$.

Die wichtigen Impulse erkennend, die von solchen Kongressen und Tagungen ausgingen, motivierten weitere derartige Veranstaltungen auch anderen Ortes. Im Jahre 1930 beteiligten sich angesehene ausländische Gäste wie Dirac, Pauli, Sommerfeld u. a. an einem Kongreß in Odessa ${ }^{34}$. Seit 1929 fanden alljährlich Zusammenkünfte einer kleineren von Bohr geladenen Gruppe von Theoretikern in Kopenhagen statt ${ }^{35}$. Reich besucht waren auch die von Scherrer und Pauli organisierten physikalischen Vortragswochen in Zürich ${ }^{36}$ und die von Debye und Heisenberg ins Leben gerufenen Veranstaltungen in Leipzig ${ }^{37}$. Der Kernphysik-Kongreß 1931 in Rom ${ }^{38}$, der Elektrizitätskongreß 1932 in Paris ${ }^{39}$ und der internationale Physikerkongreß 1934 in London ${ }^{40}$ waren weitere herausragende Ereignisse dieser Zeit.

Auch in Amerika veranstaltete man jetzt häufiger derartige Treffen mit internationaler Beteiligung. Seit 1926 fanden in Ann Arbor in Michigan regelmäßig die sogenannten Sommerschulen statt, zu denen in der ersten Zeit vorwiegend ausländische Gäste zu theoretischen Vorträgen eingeladen wurden ${ }^{41}$. Die Verbindung zu Europa hielten insbesondere die beiden 


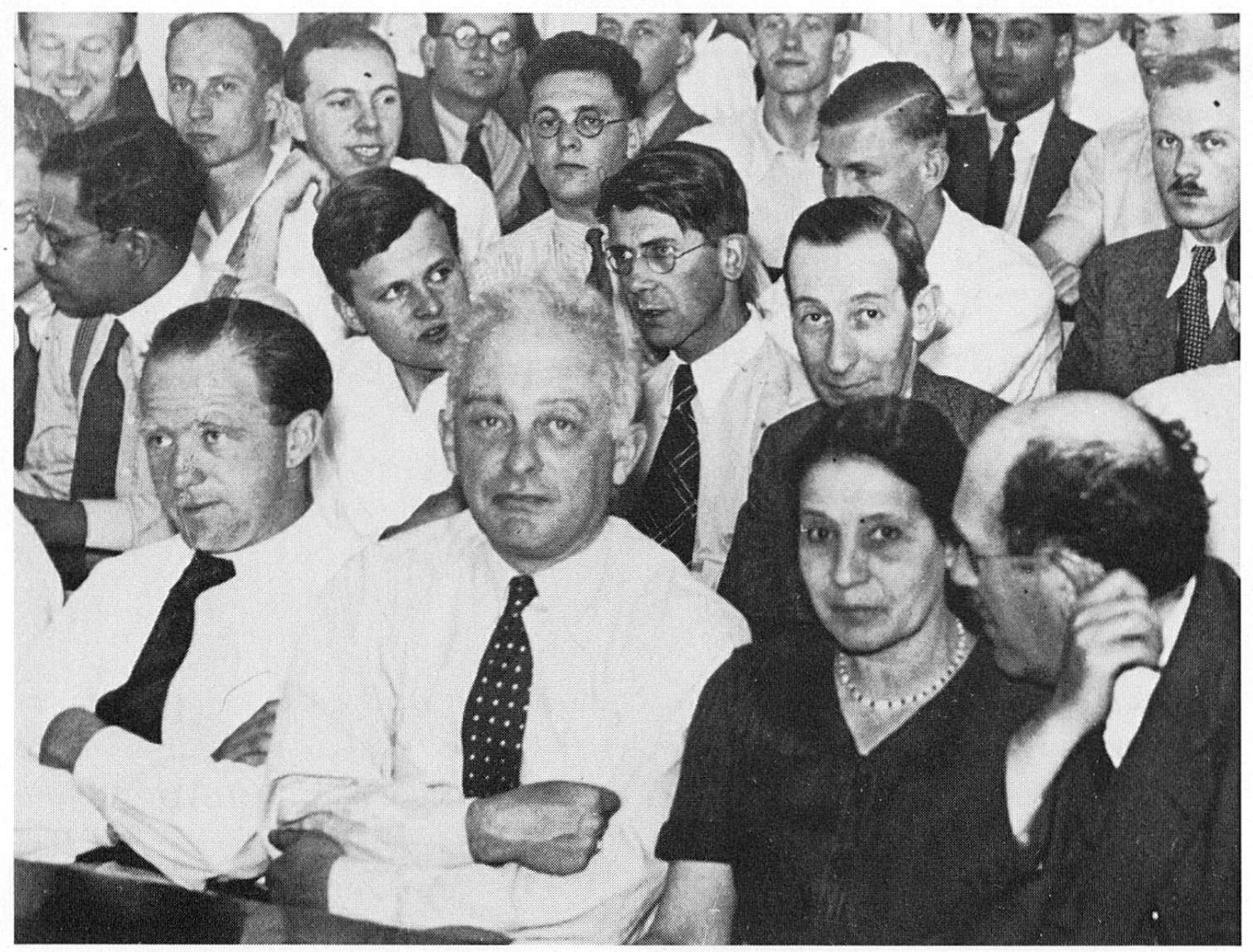

Abb.2. Physikertreffen in Kopenhagen. In der ersten Reihe (von links nach rechts): Heisenberg, ?, Meitner und Stern. Zweite Reihe: ?, v. Weizsäcker, Hund, ?. Rechts hinten Dirac

genannten Ehrenfest-Schüler Goudsmit und Uhlenbeck aufrecht. Diesem Zwecke dienten auch längere Europareisen amerikanischer Gelehrter und Forscher wie Millikan, Randall und des sich inzwischen in Amerika heimisch fühlenden Goudsmit.

Alle diese Bestrebungen zusammen würde schon hinreichen, um das schnelle Wachstum und die Verlagerung des Schwerpunktes auch der theoretischen Forschung von Europa nach Amerika während der dreißiger Jahre zu erklären ${ }^{42}$. Nicht daraus verstehen kann man dagegen den rapiden Niedergang dieses Forschungszweiges in einigen europäischen Ländern wie Deutschland und Italien, die offenbar die idealsten Voraussetzungen für eine kontinuierliche Weiterentwicklung boten.

Schon in den zwanziger Jahren kam es an einigen Fakultäten vor, daß einzelne Forscher in Deutschland und in einigen anderen Ländern wegen 
ihrer jüdischen Herkunft nur mit Mühe eine Position erhalten konnten. Für die Nachfolge von Max Born in Frankfurt wurden z. B. zwei so ausgezeichnete Gelehrte wie Otto Stern und Paul Epstein aus solchen Motiven abgelehnt ${ }^{43}$, was letzteren schließlich zur Auswanderung veranlaßte. Nur mit Mühe konnte der anerkannte Spektroskopiker Friedrich Paschen sich einen Theoretiker wie Landé nach Tübingen holen, «obwohl er Jude ist», wie es ein Zeitgenosse unverblümt ausdrückte ${ }^{44}$. Ähnliche Erfahrungen machte Einstein in Berlin, als es galt, für den Physiker-Philosophen Hans Reichenbach eine Stelle zu finden. «Planck und Laue haben sich bemüht», heißt es in seinem Schreiben vom 10. Juni 1926, «ihm [Reichenbach] eine Lehrstelle an der Berliner Universität zu verschaffen. Dies scheitert aber ziemlich an der politischen Sache, die mitzuteilen für ein Nicht-Preußen-Ohr zu blöde wäre.»

Dennoch war es keineswegs die Regel, wie die Besetzung vieler anderer Lehrstühle beweist.

Erst mit der Machtübernahme im Frühjahr 1933 durch die Nationalsozialisten beginnt die systematische Verdrängung jüdischer und system-

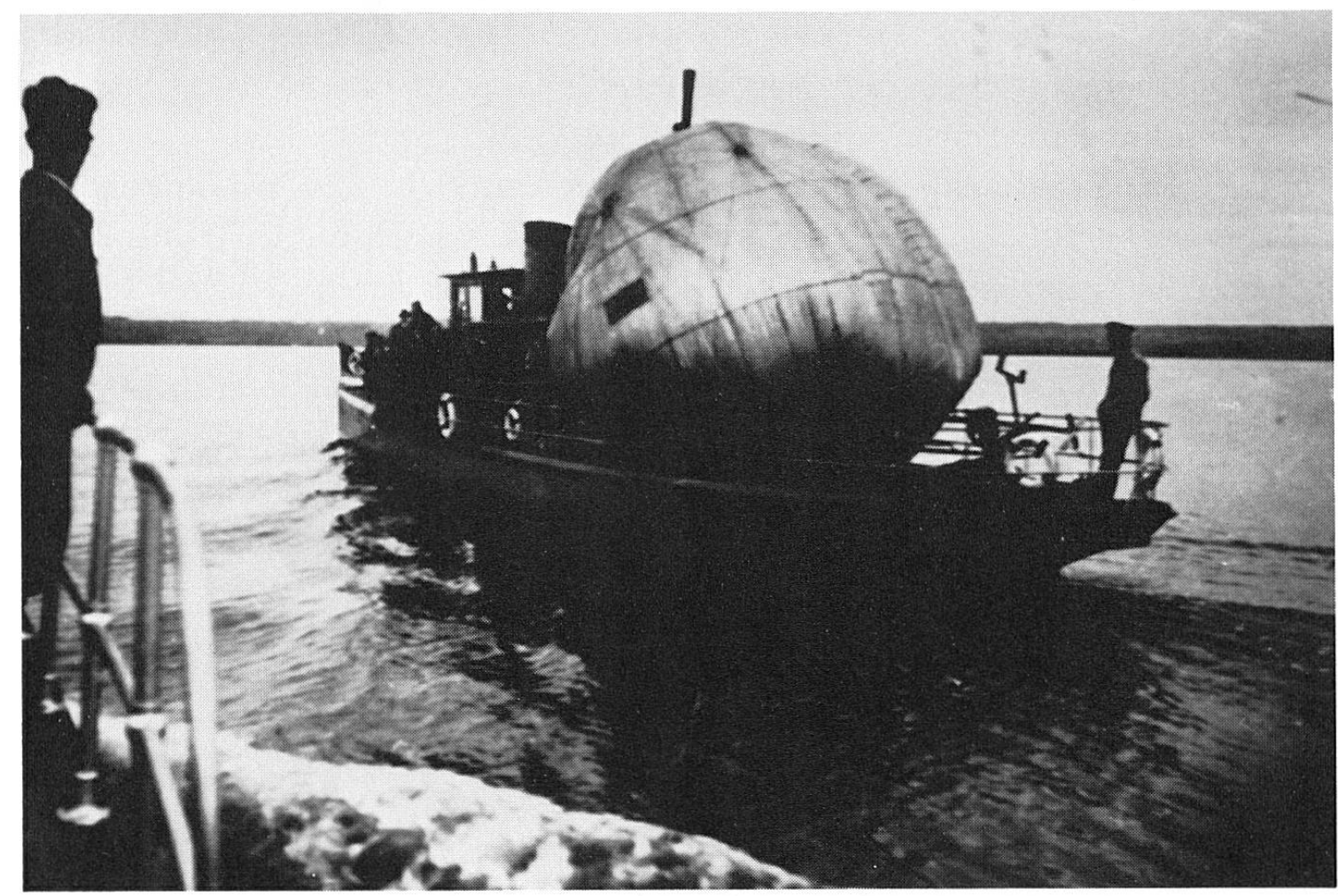

Abb.3. Erich Regners Forschungsschiff «Undula» bei der Messung der Höhenstrahlung in großen Tiefen auf dem Bodensee 
feindlicher Wissenschaftler aus Deutschland ${ }^{45}$. Einsteins protestvoller Austritt aus der preußischen Akademie der Wissenschaften signalisierte nur den Anfang.

Durch das Gesetz zur Wiederherstellung des Berufsbeamtentums sind in erster Linie nur die bereits beamteten Forscher erfaßt. Aber auch die jüngeren Nachwuchskräfte, unter ihnen ein großer Teil der zukünftigen wissenschaftlichen Elite, konnte keine angemessene Stellung finden ${ }^{46}$. Eine Liste der davon betroffenen Personen spricht für sich selbst. Insgesamt waren es etwa 150 ausgebildete Physiker, die das Land verließen.

In die durch die Entlassungen frei gewordenen Positionen rückten nun meist minderqualifizierte Sympathisanten des Systems. Politische und ideologische Eingriffe erschwerten selbst diesen die Fortsetzung der Forschungsarbeit.

Eine Schlüsselstellung bei der Vermittlung neuer Positionen nahm der seit 1912 als Nachfolger von Lorentz in Leiden wirkende Ehrenfest ein. Er verfügte bereits über hinreichende Erfahrungen aus der Zeit, als er sich um das Schicksal der nach der Revolution verbannten Wissenschaftler darunter über 200 Professoren - zu kümmern hatte. Seine späteren Reisen nach Amerika hatten das Ziel, «persönliche Kontakte zu Gunsten der jüngeren holländischen und russischen Physiker zu kriegen», wie er am 9. September 1922 Epstein mitteilte.

Zusammen mit so einflußreichen Physikern wie Einstein und Born organisierte Ehrenfest jetzt rasch eine Hilfsorganisation im Auslande, die es sich zur Aufgabe machte, neue Positionen für die durch das NS-Regime Vertriebenen zu beschaffen.

Wenn diese Forscher in den meisten Fällen auch nur einen geringen Teil ihrer Habe mit sich führen durften, die geistige Substanz ihrer Wissenschaft ist mit ihnen in die Emigration gegangen. J. J. Thomson eröffnete 1936 in Oxford eine Tagung mit dem Hitlergruß. Die Verblüffung der Teilnehmer zerstreute sich, als er erklärend hinzufügte: Dank Hitler haben wir heute Franz Simon, Kurt Mendelssohn, Rudolph Peierls und viele andere unter uns ${ }^{47}$.

Der Zuwachs, den diese Länder durch die Aufnahme der Emigranten für ihre Wissenschaft erhielten, wird deutlich, wenn man die personelle Besetzung vieler amerikanischer und englischer Forschungsstätten dieser Zeit überprüft. In Princeton scharte sich eine ansehnliche Gruppe um Einstein. Besonders die nun in Amerika aufblühende Kernphysik gewann mit Hans Bethe, Eugen Wigner, Eduard Teller, Leo Szilard, Felix Bloch und Victor 


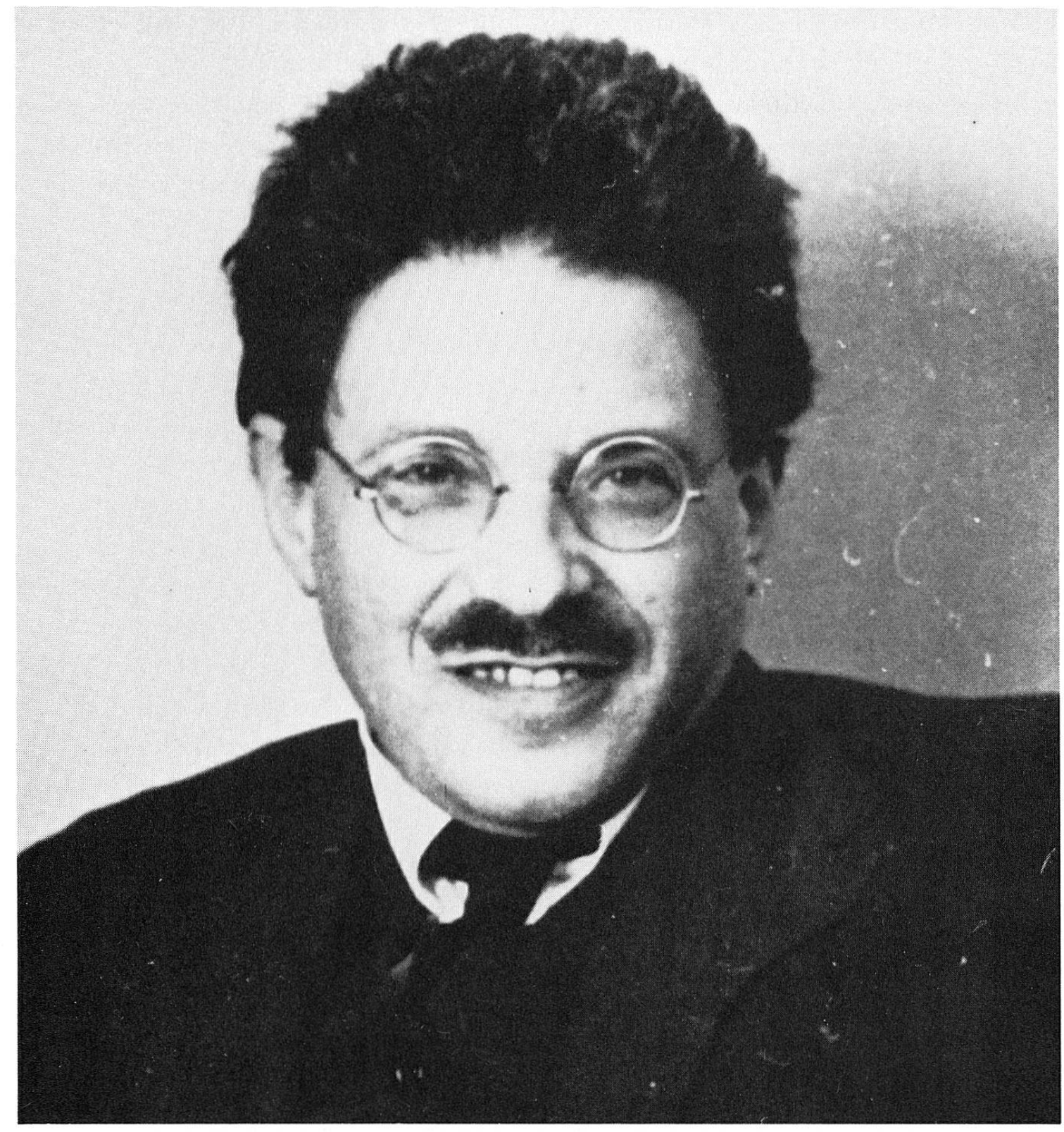

Abb.4. Paul Ehrenfest, kurz vor seinem Tode im September 1933 aufgenommen

Weißkopf an der Spitze einen entscheidenden Auftrieb. Schon bald sind die Erfolge auf diesem Sektor so groß, daß Heisenberg mit Anerkennung feststellen mußte: «Bei der Kernphysik in Amerika können wir hier gewissermaßen nur mit Bewunderung und ein wenig Neid zusehen.» Ein zweiter Aderlaß für die Wissenschaft folgte 1938 auf die Okkupation von Österreich durch die deutschen Truppen und die sog. «Reichskristallnacht» in Deutschland. Auch diesmal ließen sich rasch Interessenten im Ausland für die 
bekannteren unter den vertriebenen Forschern finden. Für die weniger eminenten bedeutete es öfter das Ende ihrer Laufbahn.

Auch das faschistische Italien blieb von dieser Entwicklung nicht verschont, als Mussolini, dem deutschen Beispiel folgend, auch dort die Rassengesetze einführt. Am 1. August 1938 berichtet Goudsmit von einer Europatournée seinem Kollegen Randall: "If Italy follows the german example, some valuable man might become available ... If they go as far as Germania, finally also Fermi." Wenig später ist es bereits soweit. Am 1. September 1938 schreibt Fermi aus Lugano: "But despite my natural optimism I must confess, that I expect rather difficult times ... in case there should be in America a convenient position for me, I would gladly accept it ..." Das Schicksal des durch Fermis Bemühungen in Italien soeben erst ins Leben gerufenen Forschungszweiges war damit besiegelt.

Aber nicht allein die Verdrängung von Wissenschaftlern behinderte den Forschungsbetrieb. 1934 wurde der Präsident der Notgemeinschaft Friedrich Schmidt-Ott entlassen und durch Johannes Stark ersetzt. Bekannt durch seine Theoriefeindlichkeit, versagte Stark jetzt theoretischen Arbeiten prinzipiell jegliche Unterstützung durch die Notgemeinschaft. Das Führerprinzip sollte fortan auch in der Wissenschaft maßgeblich sein. Wie man tatsächlich über Stark dachte, zeigen Äußerungen wie die des Breslauer Physikers Erwin Fues in einem Schreiben vom 2. Juli 1936 an Schrödinger: «Wir hoffen, daß uns der Erfinder des «Starkstroms» noch eine Weile ruhig an unserer theoretischen Physik läßt.»

Im gleichen Jahr 1934 publizierte Johannes Stark seine berüchtigte Schrift über «Nationalsozialismus und Wissenschaft», in der er in sehr provozierender Weise einen Zusammenhang von Rasse und Naturforschung zu konstruieren versuchte.

Weil nun gerade auf dem Gebiet der theoretischen Physik die Lehren jüdischer Gelehrter wie Einstein und Bohr vorherrschend waren, wurde die Theorie schlechthin als jüdische Wissenschaft verdammt. So gerieten jetzt auch die wenigen in Deutschland und Österreich noch zurückgebliebenen Theoretiker in Konflikt mit dem Regime. Während der Begründer der Wellenmechanik Schrödinger protestvoll nach England ausgewandert war, blieb Heisenberg im Lande und nahm den Kampf mit den Theoriegegnern auf ${ }^{48}$. Ladenburg, der ihn im Sommer 1937 aufsuchte, berichtete: «Heisenberg war sehr optimistisch und meinte, er werde Stark schon unterkriegen.»

Der Schaden, den diese Maßnahmen der deutschen Wissenschaft zufügten, wurde auch im Ausland verständnislos verfolgt. In einem Schreiben von 
Goudsmit an den englischen Physiker Charles Galton Darwin vom 23. November 1933 heißt es: "The situation in Germany is indeed deplorable. However, since they have kicked out all the best scientists and since those who were not kicked out left out of free-will, they soon will be a nation of the 5 th rank as far as scientific culture is concerned ..."

Weiter sprach Goudsmit seine Hoffung aus, ob nun nicht auch Heisenberg dem Beispiel von Schrödinger folgen würde. In einem Brief vom 9. Januar 1934 äußerte sich hierzu Heisenberg: «Sie können sich denken, daß auch mir der Zustand der theoretischen Physik in Deutschland und das Wohl der Menschen, die sie vertreten, große Sorgen macht. Man muß eben versuchen, unter den gegebenen Umständen das Beste zu erreichen ...»

Heisenbergs Entschluß, trotz der gegen ihn einsetzenden Hetzkampagne der Nationalsozialisten in Deutschland zu bleiben und Widerstand zu leisten, wurde im Ausland mißverstanden und hat ihn in den Ruf gebracht, dem «3. Reich» nicht ganz ablehnend gegenüberzustehen ${ }^{49}$. Aus heutiger Sicht gesehen war sein Verbleiben von entscheidender Bedeutung für die spätere Entwicklung. Allein einem Forscher von seinem Range war es möglich, im «3. Reich» und unter der Gegnerschaft vieler Kollegen die theoretische Physik am Leben zu erhalten. Als die Alliierten über die Neuordnung der deutschen Nachkriegswissenschaft berieten, wurde in einem Gutachten darauf hingewiesen, daß: "... as soon as given a chance, Heisenberg will do all that he can to rethrengthen german science in order that it, and consequently Germany, can again play a dominating role in the world ..."

Die Angriffe gegen die von Stark als dogmatisch bezeichnete theoretische Physik mußten schließlich nach dem sog. «Münchener Religionsgespräch» im Jahre 1940 eingestellt werden. Auf diese Weise war es möglich gewesen, wenigstens eine kleine Schar von theoretisch geschulten Physikern zu erhalten, die nach der Katastrophe den Wiederaufbau aufnehmen konnten.

$\mathrm{Da}$ nicht allein in den faschistischen Ländern die Wissenschaft der 30er Jahre durch ideologische Forderungen eingeschränkt und behindert werden konnte, zeigen die Ereignisse im Sowjetstaat. In der auf der neuen Physik fußenden Philosophie des Neopositivismus, wie sie besonders von Hans Reichenbach, Philipp Frank und Moritz Schlick vertreten wurde, sahen die Stalinisten einen bewußten Angriff gegen den Marxismus. Selbst Einstein blieb von ihnen nicht verschont, und seine Relativitätstheorie wurde zeitweilig verboten, weil er entgegen der leninistischen Auffassung wissenschaftliche Erkenntnisse auch ohne Empirie für möglich hielt. Desgleichen 
wurden die Folgerungen aus der Heisenbergschen Unschärferelation - und mit ihr auch seine Matrixmechanik - abgelehnt, weil sie eine Einschränkung der von der materialistischen Weltanschauung als grundlegend angesehenen Kausalität bedeutet hätten.

In der Folge fanden zahlreiche Auseinandersetzungen zwischen den russischen Philosophen und Physikern statt, die im Juni 1934 auf einem Kongreß der wissenschaftlichen Akademie mit den Vorträgen von Joffé und Vavilow ihren Höhepunkt erreichten ${ }^{50}$.

Auch hier setzte sich das System zur Wehr, indem es die ihm ungenehmen Wissenschaftler entließ oder verhaftete ${ }^{51}$. Wolfgang Pauli, der im September 1937 einen Kernphysikerkongreß in Leningrad und Moskau besucht hatte, berichtete am 21. August 1938 über die neuesten ihm zugegangenen Nachrichten aus Rußland: «[In Charkow] sind die Hälfte der Mitglieder verhaftet, u. a. Schubnikow, Rosienkiewicz, Houtermans. Zu den sonst in Rußland verhafteten gehört neuerdings auch der Mathematiker F. Noether, ...» Obwohl diese Auswüchse weniger bekannt wurden und auch nicht so katastrophale Ausmaße wie in Deutschland annahmen, so haben sie sicher auch der Sowjetwissenschaft schweren Schaden zugefügt. Äußerst störend wirkte sich zudem die Einschränkung der in den 20er Jahren noch gewährten Freizügigkeit sowjetischer Gelehrter auf den Transfer von Wissenschaft aus ${ }^{52}$.

Obwohl wir in dieser Darstellung vorwiegend ideologische Auseinandersetzungen und politische Maßnahmen für die einschneidenden Wandlungen in der Wissenschaft der 30er Jahre verantwortlich gemacht haben, so darf doch nicht vergessen werden, daß es natürlich auch noch andere Ursachen für diese Verschiebungen gibt, die in der Natur der Sache selbst liegen.

Selbst bei der hohen Spezialisierung der modernen Naturwissenschaft ist eine vollständige Trennung von Theorie und Experiment undenkbar. Dadurch bleibt aber die Entwicklung der Theorie stets an den Stand der Experimentalwissenschaften gebunden. Der mit zunehmender Kenntnis einhergehende experimentelle Aufwand führte jedoch zu immer kostspieligeren Großforschungsanlagen, die nicht mehr auf europäische Verhältnisse zugeschnitten waren. Der früher noch im Vordergrund stehende Einzelforscher wird immer mehr durch Teamarbeit ersetzt. Für eine Forschung dieses Stils waren aber Amerika und Rußland viel besser gerüstet als das traditionsgebundene Europa. Diese Lücke wurde erst durch die europäische Zusammenarbeit bei der Großforschung nach dem Kriege geschlossen.

Abschließend kann man also sagen, daß der komplexe Prozeß des rapiden wissenschaftlichen Aufstiegs von Amerika und der Sowjetunion ohne 
Zweifel eine autonome Entwicklung im Gefolge ihres politischen und wirtschaftlichen Wachstums gewesen ist, für welches das Zusammenspiel von Wissenschaft und Technik Voraussetzung sind. Diese Entwicklung wurde jedoch durch die politischen Ereignisse in Europa der 30er Jahre entscheidend beschleunigt. Um so einschneidender waren die Auswirkungen auf die Naturwissenschaften und insbesondere auf die Physik im deutschen Sprachbereich. Die theoretische Physik, die hier im Zusammenspiel mit der Mathematik einst eine führende Rolle gespielt hatte, war für viele Jahre auf das Niveau einer "Nation of the 5 th rank" abgesunken.

\section{Anmerkungen}

${ }^{1}$ Vgl. hierzu W.Gentner: «Individuelle und kollektive Erkenntnissuche in der modernen Naturwissenschaft.» Freiburger DIES Universitas 9, 1-16 (1961/62). - W.Bothe: «Der Physiker und sein Werkzeug.» Vorträge und Schriften der Preußischen Akademie der Wissenschaften. Heft 22. Berlin 1944. - Die Abgrenzung von «Theorie und Experiment in der exakten Naturwissenschaft» versuchte W.Gerlach in einem Vortrag auf der Naturforscherversammlung im September 1936 in Dresden zu umreißen. Vgl. Naturwiss.24, 721-741 (1936). - Den Einfluß, den die europäischen Einflüsse auf die Entwicklung der amerikanischen Physik ausgeübt haben könnte, untersucht u.a. J.Heilbron: «Physics in the United States just before Quantum Mechanics.» Vortrag in Lecce (Vgl. Fußnote 15) H.B. G. Casimir: «Gedanken über die Zukunft der Physik.» Naturwiss.44, 245-248 (1957).

${ }^{2}$ Immanuel Estermann: "History of molecular beam research: Personal reminiscences of the important evolutionary period 1919-1933." American J. Phys. 43, 661-671 (1975).

${ }^{3}$ Einen zeitgenössischen Überblick über die Entwicklung der Kernphysik der 30er Jahre findet man in den Berichten von G. Hoffmann und R. Fleischmann: «Atomkerne, Radioaktivität 1. u. 2.» In: Die Physik 3, 109-132 (1935), 8, 17-32 (1940); A. Flammersfeld: «Zur Geschichte der Atomkernisomerie.» In: «Beiträge zur Physik und Chemie des 20. Jahrhunderts. Lise Meitner, Otto Hahn und Max v. Laue zum 80. Geburtstag.» Braunschweig 1959, dort S. 74-77. - Eine zusammenfassende historische Darstellung der Theorie findet man bei D.M.Brink: «Kernkräfte. Einführung und Originaltexte.» Braunschweig 1971. - Interviews, Diskussionen, Erinnerungen und Darstellungen von beteiligten Forschern zur Kernphysik in: Ch. Weiner und E.Hart (ed.): "Exploring the History of Nuclear Physics." New York 1972; R. Stuewer: "Nuclear Physics in Retrospect. Proceedings of a Symposium on the 1930's." Minneapolis 1979.

${ }^{4}$ E.Regener: «Die kosmische Ultrastrahlung.» Naturwiss. 25, 1-11 (1937). - E. Miehlnickel: «Höhenstrahlung.» Dresden/Leipzig 1938. - V.Mukherji: "A History of the Meson Theory of Nuclear Forces from 1935 to 1952." Archive for Hist. Exact Sci. 13, $27-102$ (1974). H. Thirring: «Der gegenwärtige Stand unserer Kenntnisse von der kosmischen Strahlung.» Wiener Vorträge, vierter Zyklus, Wien 1939.

${ }^{5}$ Herbert Fröhlich berichtete, daß er damals mit Hans Bethe wettete, wer zuerst den Fehler in einer der zahlreich erscheinenden theoretischen Arbeiten über Supraleitung finden würde. Vgl. z.B. H.Bethe und H.Fröhlich: «Magnetische Wechselwirkung der Metallelektronen. 
Zur Kritik der Theorie der Supraleitung von Frenkel.» Z. Phys. 85, 389-397 (1933). M.v.Laue: «Geschichtliches über Supraleitung.» Forschungen und Fortschritte 25, 279-281 (1949). - P. Debye: «Der Vorstoß zum absoluten Nullpunkt.»Umschau 40, $41 \mathrm{ff}$. (1936).

${ }^{6}$ Siehe hierzu H.Welker: "Impact of Sommerfeld's work on solid state research and technology." In: F.Bopp und H.Kleinpoppen: "Physics of the One- and Two-Electron Atoms." Amsterdam 1969, dort S.32-43. - H.B.G.Casimir: "Pauli and the Theory of the Solid State." In: M.Fierz und V.F.Weißkopf (eds.): "Theoretical Physics in the Twentieth Century." New York 1960.

7 W.Bothe: "The coincidence method." Nobel-Lecture 1954. - «Kernphysikalische Arbeiten aus dem Institut für Physik am Kaiser-Wilhelm-Institut für medizinische Forschung, Heidelberg.» Z.f. technische Physik 19, 565-569 (1938). - R. Fleischmann: «Zur Entdekkungsgeschichte der künstlichen Kern- $\gamma$-Strahlung.» Naturwiss. 38, 465-467 (1951).

8 C.D. Anderson: "The Production and Properties of Positrons." Nobel-Lecture 1936. - Vgl. hierzu auch die Darstellung von N.R. Hanson: "The Concept of the Positron. A philosophical analysis" und die kritischen Bemerkungen von R. Peierls in seiner Buchbesprechung in Hist. of Sci. 4, 124-129 (1965).

9 J.Chadwick: "The Existence of a Neutron." Proc. Roy. Soc. A. 136, 692-708 (1932). J.Bromberg: "The Impact of the Neutron." Hist. Stud. Phys. Sci. 3, 307-341 (1971). E.P. Wigner: "The Neutron: The Impact of Its Discovery and Its Uses." In: R. Stuewer, op. cit. (Fußnote 3), dort S. 157-173. - W.D. Harkins: «Neuton und Neutron, das Element mit der Ordnungszahl Null.» Naturwiss. 21, 575-577 (1933).

${ }^{10}$ Vgl. hierzu H.A.Bethe: "The Happy Thirties." In: R. Stuewer, op. cit. (Fußnote 3), dort S.11-26. - W. Heisenberg: «Prinzipielle Fragen der modernen Physik.»Wiener Vorträge, dritter Zyklus, Wien 1936. - O. Frisch: "What little I remember." Cambridge 1979.

11 W. Pauli: «Zur älteren und neueren Geschichte des Neutrinos.» In: W.Pauli: «Aufsätze und Vorträge über Physik und Erkenntnistheorie.» Braunschweig 1961, dort S.156-180.

${ }^{12}$ Dieser Brief aus dem Nachlaß von P.S.Epstein befindet sich in der Millikan Library des Caltec in Pasadena. - Epsteins Berufung nach Amerika wurde von seinen europäischen Kollegen aufmerksam verfolgt. A.Rubinowicz, ebenfalls ein Sommerfeld-Schüler, der damals nach einer neuen Position Ausschau hielt, schrieb ihm am 4. Juni 1922 von Wien aus: «Schon allein die Tatsache, daß Sie - ohne Ihnen schmeicheln zu wollen - der erste theoretische Physiker in Amerika sind, wird die besten Köpfe unter den amerikanischen Studenten in Ihren Wirkungsbereich bringen ...» Sommerfeld hatte um diese Zeit eine Einladung nach Amerika angenommen. Er erkundigte sich am 29. Juni 1922 bei seinem Schüler: «Ich würde ja gewiß gern die Märchenwelt des Mt. Wilson bewundern und die erstklassige Forschungsanstalt, die die Energie des Herrn Millikan in Pasadena geschaffen hat ... Aber es ist klar, daß ich mich als Deutscher nicht anbieten werde ...» Die letztere Bemerkung bezieht sich auf die weiterhin anhaltende Ablehnung gegenüber den deutschen Wissenschaftlern nach dem Ersten Weltkrieg, die erst allmählich abklang, nachdem Deutschland in den Völkerbund aufgenommen wurde. Der hier noch sehr selbstbewußte Ton des europäischen Physikers ist Ende der 30er Jahre gänzlich verschwunden. In einem Schreiben vom 1.Januar 1939 an Pauli äußerte Sommerfeld melancholisch: «Vielleicht liegt die Zukunft der Kultur nicht (auf dem Wasser), sondern jenseits des großen Wassers.» - Paul Ehrenfest erfaßte die sich ändernde Situation schon viel früher. In einem Brief vom 
10. Januar 1924 aus Pasadena an seine beiden damaligen Schüler Goudsmit und Tinbergen schrieb er: «Und stets beschäftigt mich die Frage: Wird West-Europa in 20, 30 Jahren irgend etwas leisten, was dann die U[nited] St[ates] nicht besser leisten werden? - Diese Frage ist natürlich von der anderen zu trennen: Wird Amerika etwas leisten, was dem Größten, Tiefsten und Besten zu vergleichen ist, was West-Europa geleistet hat? ...» (Original des Briefes in der Goudsmit-Collection, American Institute of Physics, New York). - Siehe hierzu auch M.J.Klein: "America Observed: Paul Ehrenfest's visit in 1923/24." Vortrag auf der "Conference on the Recasting of Science between the Two World Wars." Florenz/Rom 1980.

${ }^{13}$ Die ersten Kontakte mit Harrison M. Randell, dem Leiter des physikalischen Instituts in Ann Arbor, hatte Ehrenfest hergestellt. Wie sehr sich Ehrenfest um das Schicksal seiner Zöglinge kümmerte, zeigen folgende Zeilen aus einem Brief vom 7.Dezember 1926 an Randall: «... Aber man verlange von ihnen [Uhlenbeck und Goudsmit] keine wissenschaftlichen Funde. Sie werden natürlich darüber lächeln ..., aber ich formuliere damit sicher eine wichtige Sorge der besten jungen Leute. Inmitten der schrecklichen Hast, mit der jetzt überall publiziert wird, scheint ja das, was das eigentliche Wesen der theoretischen Physik ausmacht: Der Wunsch, durch ruhiges Nachdenken und Diskutieren mit Freunden die Physik besser zu begreifen; dieser Wunsch scheint als ein unerlaubter undemokratischer Luxus getadelt zu werden. Das holländische Wesen liebt nicht die Hast und sehnt sich vor allem nach diesem ruhigen tiefen Nachdenken ...»

${ }^{14}$ Biographische Angaben über A.Landé findet man in der Festschrift "Perspectives in Quantum Theory", herausgegeben von W. Yourgrau und A.van der Merwe. Cambridge 1971.

${ }^{15}$ Eine historische Darstellung mit vielen bibliographischen Angaben über die Entwicklung der neueren Physik in Amerika findet man bei D. J. Kevles: "The Physicists. The History of a Scientific Community in Modern America" New York 1977. - Siehe auch R. Seidel: "Institutional Aspects of the Transmission of Quantum Mechanics to the United States." Workshop: "The Growth of Quantum Mechanics in the 20's and the Cultural, Economic and Social Context of the Weimar Republic and of the USA." Lecce, 3.-6. September 1979.

${ }^{16}$ Robert Oppenheimer war zuerst 1925/26 bei J. J. Thomson in Cambridge. Er promovierte 1927 bei Born in Göttingen. Anschließend kam er nochmals 1928/29 zu Ehrenfest nach Leiden und zu Pauli nach Zürich. Über R. Oppenheimer gibt es zahlreiche Untersuchungen. Wohl am aufschlußreichsten ist der vor kurzem von Alice Kimball Smith und Ch. Weiner herausgegebene Briefwechsel "Robert Oppenheimer. Letters and Recollections." Cambridge, Mass. 1980.

17 John Slater war ebenso wie Oppenheimer ein Harvard-Schüler. Nach seiner Promotion bei P.W.Bridgman ging er 1923/24 nach Cambridge und Kopenhagen. Dort lieferte er Bohr mit seinen Vorstellungen wichtige Anregungen, die zu der berühmten Strahlungstheorie führten. 1929 war er nochmals bei Heisenberg in Leipzig. Seinen Werdegang hat Slater in seinem autobiographischen Werk: "Solid-State and Molecular Theory: A Scientific Biography" New York, London, Sydney, Toronto 1975, beschrieben.

18 Der aus Galizien mit seinen Eltern nach Amerika eingewanderte Isidor Rabi erwarb 1927 den Doktorgrad an der Columbia-University und war von 1927-1929 nacheinander an den Universitäten Zürich, München, Kopenhagen, Hamburg, Leipzig und zuletzt wieder in Zürich. Biographische Angaben findet man in Jeremy Bernstein: "Experiencing Science. 
Profiles in Discovery." New York 1980, und D.J.Kevles, op. cit. (Fußnote 15), dort S. $213 \mathrm{ff}$.

${ }^{19}$ Auch Gregory Breit ist als Kind mit seinen Eltern aus Rußland nach Amerika eingewandert. Nach abgeschlossenem Studium war er 1921/22 bei Ehrenfest in Leiden. 1928 kam er nochmals nach Europa und besuchte u. a. Pauli in Zürich.

${ }^{20}$ David M. Dennison promovierte 1924 an der Universität in Michigan bei Oskar Klein (der dort von 1923-1925 gastierte) und W.F. Colby. Von 1924-1927 hielt er sich nacheinander in Zürich, München, Kopenhagen, Cambridge und Leiden auf. Weitere Angaben im Nachruf von H.R. Crane und K.T. Hecht in Physics Today, Juli 1976, S. 71.

${ }^{21}$ Zwei Jahre nach seiner Promotion bei Karl T.Compton an der Universität Princeton besuchte Carl Eckart 1927/28 München, Berlin und Göttingen. Bereits 1926 hatte er unabhängig von Schrödinger und Pauli die Äquivalenz von Quanten- und Wellenmechanik nachgewiesen.

${ }^{22}$ Eugene Feenberg besuchte schon vor seinem Studienabschluß 1933 einige europäische Universitäten, darunter München, Zürich, Rom, Leipzig und Berlin.

${ }^{23}$ Edwin C. Kemble aus Harvard war 1927 in München und Göttingen.

${ }^{24}$ William V. Houston aus Pasadena war 1927/28 in München.

${ }^{25}$ Ralph Kronig war schon als Kind mit seinen Eltern von Dresden nach New York gezogen. An der Columbia University erwarb er 1924 den Doktorgrad. Anschließend kam er mit einem Rockefeller-Stipendium nach Europa. U.a. war er in Kopenhagen bei Bohr und in Zürich bei Pauli. Später wirkte er für viele Jahre in Holland. Kronig ist einer der wenigen Stipendiaten, die nicht wieder nach Amerika zurückkehrten.

${ }^{26}$ Robert S. Mulliken hielt sich vorübergehend in Europa auf.

${ }^{27}$ Nach Abschluß seines Studiums 1925 kam Linus Pauling für zwei Jahre nach München, Zürich und Kopenhagen, bevor er wieder nach Pasadena zurückkehrte.

28 John H.van Vleck erwarb 1922 den Doktor bei Kemble in Harvard und besuchte im Sommer 1923 Kopenhagen. Ebenso nahm er 1930 als einziger Amerikaner am 6. Solvay-Kongreß teil. Bei dieser Gelegenheit besuchte er auch Werner Heisenberg in Leipzig. Siehe hierzu auch seinen Artikel "American Physics Comes of Age" in Physics Today, Juni 1964, dort S.21-26.

${ }^{29}$ Earle Kennard war im WS 1926/27 in Göttingen. Weiter wäre noch zu nennen Edward Condon, der sich 1927 in Göttingen und München aufhielt und hier das nach ihm und James Franck benannte Franck-Condon-Prinzip der Molekültheorie aufstellte.

${ }^{30}$ George Gamow ging 1928 zuerst nach Göttingen, dann nach Cambridge und Kopenhagen. Nach kurzer Lehrtätigkeit in Leningrad 1931/33 verließ er Rußland definitiv und übernahm schließlich eine Professur in Washington. Einige Angaben hierzu findet man in seiner autobiographischen Schrift: "My World Line" New York 1970, N, dort S.48ff. - Lev Davidovich Landau besuchte 1929/31 u.a. Kopenhagen, Zürich, Göttingen, Leiden und Cambridge. - Jakov Frenkel war im WS 1925/26 in Berlin, Hamburg und Göttingen. Vladimir Fock arbeitete im Frühjahr 1928 bei Born in Göttingen. Viele seiner Arbeiten publizierte er (bis 1933) in der Zeitschrift für Physik. - Weitere Angagen über russische Physiker findet man bei A.F. Joffé: «Begegnungen mit Physikern.» Basel 1967.

${ }^{31}$ Siehe hierzu F.Hund: «Göttingen, Kopenhagen, Leipzig im Rückblick.» In: «Werner Heisenberg und die Physiker unserer Zeit.» Braunschweig 1961, dort S. 1-7. - P.Robertson: “The Early Years. The Niels Bohr Institute 1921-1930.” Kopenhagen 1979. 
${ }^{32}$ E. Amaldi: "Personal Notes on Neutron Work in Rome in the 30's and Postwar European Collaboration in High-Energy Physics." In: "Proceedings of the Int. School of Physics 'Enrico Fermi'." Varenna on lake Como, Villa Monasterio. 31. Juli-12. August 1972. New York/London 1977, dort S.294-351. - G.Holton: "Fermi's group and the recapture of Italy's place in physics." In: "The scientific imagination. Case studies." Cambridge 1978, dort S.155-198. - E.G.Segré: "Nuclear Physics in Rome." In: R.H.Stuewer, op. cit. (Fußnote 3), dort S.35-62. - E. G. Segré: "Enrico Fermi, Physicist." Chicago/London 1970.

${ }^{33}$ Vgl. hierzu J. Mehra: "The Solvay Conferences on Physics." Dordrecht-Boston 1975. W.Gerlach: «Der Solvay-Kongreß 1930.» Metall-Wirtschaft, Wissenschaft und Technik. 9, 939-942, 965-1006 (1930). - Auf der 6. Konferenz 1930 wurde der Magnetismus, auf der 7.Konferenz 1933 die Atomkerne behandelt. Die 8. Konferenz über Elementarteilchen sollte im Herbst 1939 stattfinden, mußte aber wegen Kriegsausbruchs um 9 Jahre verschoben werden.

${ }^{34}$ Der nun schon fast zwei Jahrzehnte in Leiden wirkende Ehrenfest war bei der Herstellung auch dieser Verbindung zu Rußland sehr hilfreich. In einem Schreiben vom 23. Februar 1930 ersuchte er Fermi, diesmal an dem Physikerkongreß in Charkow teilzunehmen. Zur Unterstreichung der Bedeutung einer Teilnahme vermittelte er ihm den folgenden Situationsbericht:

«1. Nach dem Krieg haben in Rußland einige (in der schwersten Hungerszeit gegründete) naturwissenschaftliche Forschungsinstitute einen ganz enormen Aufschwung genommen. Ich kenne näher hauptsächlich das unter Leitung von Joffé stehende physiko-technische Institut in Leningrad sowie das unter Roshdjestwensky stehende optotechnische Institut ebendort. Bis vor kurzem konnte man solche Institute nur in Leningrad und in Moskau entwickeln. Eben jetzt aber beginnt man analoge Institute auch in Charkow und in Tomsk zu entwickeln und in den nächsten Jahren wird man trachten, dieses Netz vorzüglich eingerichteter moderner Institute weiter und weiter über Rußland auszubreiten und zwar stets in der Nähe wichtiger Industriezentren.

2. In den technisch-physikalischen Instituten wird dabei von vornherein danach gestrebt, speziell auch die theoretische und mathematische Physik sich selbständig und kräftig entwickeln zu lassen. Und da man in dieser Beziehung einige hochbegabte junge Kräfte hat (wie Frenkel, Tamm, Fock, Iwanenko, Landau u.a.), so darf man in der Tat alles Beste in dieser Beziehung erhoffen.

3. Eine der vielen Einrichtungen, die man zur Beförderung speziell der theoretischen Physik in den neuen Instituten getroffen hat, sind neben der Aussendung junger begabter Leute an ausländische Universitäten die regelmäßigen Einladungen älterer und insbesondere auch jüngerer Theoretiker aus dem Ausland ...»

${ }^{35}$ L.Rosenfeld: "Quantum Theory in 1929: Recollections from the first Copenhagen Conference." Copenhagen 1971 (Nordita reprint No.387). - "Niels Bohr in the Thirties." In: "Niels Bohr. His life and work as seen by his friends and colleagues." Amsterdam 1968, dort S. 114-136.

${ }^{36}$ Einige der «Zürcher Vorträge über Kernphysik», die vom 20. bis 24. Mai 1931 gehalten wurden, sind in der Physikalischen Zeitschrift 32 (1931) abgedruckt. - Ein zusammenfassender Bericht der Veranstaltung des Jahres 1933 über Kältephysik und Kernphysik erschien in Naturwiss. 21, 772-776 (1933). Für die Zürcher Vorträge galt der Leitsatz: «nur 
unbeschränkte Rede- und Diskussionszeit geben die Möglichkeit, Probleme ernsthaft und gründlich zu behandeln».

${ }^{37}$ Die «Leipziger Vorträge» wurden von P.Debye bei Hirzel in Leipzig herausgegeben. Die Veranstaltungen fanden unter dem Motto «Elektroneninterferenzen» (Sommer 1930), «Molekülstruktur» (1931), und «Magnetismus» (1933) statt. Die Fortsetzung scheiterte an mangelnder Unterstützung des sächsischen Unterrichtsministeriums. - Unter den Vortragenden sind: W.Kossel (Kiel), F.London (Berlin), P.A.M.Dirac (Cambridge), E.Fermi (Rom), N.F.Mott (Manchester), F.Bloch (Haarlem), R.Peierls (Zürich), F.Rasetti (Rom), G. Placzek (Leipzig), R. Kronig (Groningen), P.Kapitza (Cambridge), W.Gerlach (München), O.Stern (Hamburg), H.A.Kramers (Utrecht), H.Bethe (Tübingen), und R.Becker (Berlin).

38 «Convengo di Fisica Nucelare. Ottobre 1931 - IX.» Rom 1932.

${ }^{39}$ Auf diesem 5. Internationalen Elektrizitätskongreß gab Fermi seine berühmte Neutrinotheorie bekannt.

40 "International Conference on Physics. London 1934." Band I und II. Cambridge 1935. Ebenso erschien eine französische Übersetzung: «Congrès International de Physique organisé par l'Union Internationale de Physique et de la Physical Society, Londres 1934.» Vol.I: «L'Etat solide de la matière.» Vol.II: «Transmutations atomiques artificielles.» Vol. III: «Rayons cosmiques.» Paris 1936.

${ }^{41}$ Diese Vorträge wurden in der Regel nicht publiziert. Eine der Ausnahmen sind Fermis berühmte Vorlesungen über Quantenelektrodynamik vom Sommer 1930, die im Rev. Mod. Phys. 4, 87-132 (1932) abgedruckt wurden.

${ }^{42}$ Ehrenfest, der seinen ehemaligen Schülern Goudsmit weiterhin mit Rat und Tat unterstützte, machte (in einem Brief vom 2. Februar 1930) Vorschläge für Einladungen nach Ann Arbor: «Landé, Wigner, Pauli!!!!, Haas, Fowler, London, Heitler, Neumann, Bloch, Nordheim, Fermi!!!!!, Frenkel, Kronig.» Einige Zeit später berichtete Goudsmit: «Wir haben hier fortwährend europäischen Besuch. Vor einiger Zeit war Halpern hier. Den mochte ich nicht. Dann kamen von Laue und Ladenburg ... Jetzt ist Manneback hier aus Löwen ...» - Besondere Höhepunkte dieser Veranstaltungen in Ann Arbor waren der Besuch von Fermi im Sommer 1930 und von Pauli im Sommer 1931. Letzterer nahm trotz eines Armbruches seine Vorträge wieder auf. Da er jedoch mit seinem vergipsten Arm noch nicht schreiben konnte, half ihm Uhlenbeck und malte ihm die wichtigsten Stichworte aus seinem Manuskript vorher an die Tafel. Der Erfolg war großartig. Pauli, bekanntlich in seinen Vorträgen schwer verständlich, entfaltete jetzt im Vortrag eine Brillanz und Klarheit, die man sonst nur aus seinen Schriften kannte. (Mündliche Mitteilung von Prof. Uhlenbeck.) - Siehe hierzu auch J.H.van Vleck, op. cit. (Fußnote 28), und D.J.Kevles, op. cit. (Fußnote 15).

${ }^{43}$ Vgl. hierzu die Äußerungen Borns in seinem «Briefwechsel mit Einstein 1916-1955». München 1969, dort Brief [19] und [28].

${ }^{44}$ Sommerfeld suchte diese Tatsache in einem Brief vom 24. September 1922 an Epstein etwas abzumildern: «Was den wissenschaftlichen Export nach Amerika betrifft, so hatte ich selbst vor, Landé hierher zu empfehlen ... Daß die Tübinger Universität Landé nicht wünschte, liegt in erster Linie nicht am Antisemitismus.»

${ }^{45}$ O. Nathan und H.Norden: «A. Einstein. Über den Frieden. Weltordnung oder Weltuntergang.» Bern 1975. - A.Beyerchen: "Scientists under Hitler." New Haven/London 1977. 
(Eine Übersetzung ins Deutsche ist soeben erschienen.) - H.Mehrtens und S.Richter (Herausgeber): «Naturwissenschaft, Technik und NS-Ideologie.» Frankfurt a. M. 1980. D.C.Cassidy: «Gustav Hertz, Hans Geiger und das Physikalische Institut der Technischen Hochschule Berlin in den Jahren 1933-1945.» In: «Wissenschaft und Gesellschaft. Beiträge zur Geschichte der Technischen Universität Berlin 1879-1979.» Bd.I, Berlin/Heidelberg/New York 1979, dort S.373-387. - Ihr Schicksal haben einige Emigranten selber beschrieben. Vgl. Max Born: «Mein Leben». München 1975. - W.Elsasser: "Memoirs of a Physicist in the Atomic Age." New York/Bristol 1978.

46 A.Hermann: «50 Jahre Forschungsförderung der DFG.» Physik in unserer Zeit 2, 17-23 (1971).

47 Zitiert nach D. Nachmansohn: «German-Jewish Pioneers in Science 1900-1933.» Berlin/Heidelberg/New York 1979.

${ }^{48}$ Vgl. hierzu E. Heisenberg: «Das politische Leben eines Unpolitischen. Erinnerungen an Werner Heisenberg.» München 1980.

${ }^{49}$ Siehe hierzu die Heisenberg-Biographie von N.Mott und R. Peierls. Biogr. Mem. of Fellows of the Royal Society 23, 213-251 (1977), dort S.236.

${ }^{50}$ A. Vucinich: "Soviet Physicists and Philosophers in the 1930s: Dynamics of a Conflit." Isis $71,236-250$ (1980).

${ }^{51}$ F.Beck and W.Godin: "Russian Purge and The Extraction of Confession." London/New York/Melbourne/Sydney/Cape Town 1951.

52 Vgl. hierzu auch die Ausführungen von George Gamow, op cit. (Fußnote 30).

\section{Summary}

The climate in the Weimar Republic, notwithstanding its anti-scientific tendencies, provided excellent conditions for the development of theoretical physics. However, in a very few years this research field, which was still flourishing in the German speaking countries at the beginning of the 1930 's, was driven from its dominant position. In addition to the expulsion of the highly qualified and leading scholars by the National Socialist regime, there was a systematic obstruction of the subject; only with the greatest difficulty could the remaining theoreticians keep it alive. Whereas the two emerging world powers, the USA and USSR, were able to derive great benefit from the available reservoir of highly qualified scientists, it led to the decline of theoretical Physics in the German speaking countries in the long term. This development is documented here from numerous contemporary utterances.

Dr. Karl von Meyenn

Universität Stuttgart

Abteilung für Geschichte der Naturwissenschaften und Technik

Seidenstraße 36

D-7000 Stuttgart 1 